\title{
Aplicaciones móviles en las neurociencias: un nuevo aliado
}

Abigai Castilla- Martínez I. ${ }^{1}$, Luis Fernando Carbonell-Riquett², Yancarlos Ramos-Villegas ${ }^{3}$, Loraine Quintana-Pájaro², Luis Rafael Moscote-Salazar ${ }^{3}$

1 Estudiante de Medicina. Universidad de Cartagena, Cartagena de Indias, Colombia. Centro de Investigaciones Biomédicas (CIB), línea Cartagena Neurotrauma Research Group, Universidad de Cartagena, Cartagena, Colombia.

2 Médico. Facultad de Medicina. Universidad de Cartagena, Cartagena de Indias, Colombia. Centro de Investigaciones Biomédicas (CIB), línea Cartagena Neurotrauma Research Group, Universidad de Cartagena, Cartagena, Colombia.

3 Médico. Especialista en Neurocirugía. Faculta de Medicina, Universidad de Cartagena. Cartagena de Indias, Bolívar. Centro de Investigaciones Biomédicas (CIB), línea Cartagena Neurotrauma Research Group, Universidad de Cartagena, Cartagena, Colombia.

Rev. Chil. Neurocirugía 45: 136-139, 2019

\section{Resumen}

Gracias al desarrollo continuo de las tecnologías en telecomunicaciones, el uso de los teléfonos inteligentes se ha ido en aumento, favoreciendo el crecimiento de las aplicaciones móviles (app). Actualmente, existen más de 700.000 en las diversas plataformas, de las cuales el $3 \%$ están relacionadas con temas de salud. Las apps en salud se encuentran enmarcadas en el concepto de salud móvil o mHealth, que en la actualidad no tiene una definición clara, pero la OMS se ha referido a este como la práctica de la medicina y la salud pública soportada por dispositivos móviles. Hasta el momento existen varias revisiones sobre aplicaciones móviles en otras disciplinas médicas, la cantidad de dichas revisiones sobre apps siendo utilizadas en áreas de las neurociencias son pocas, muy a pesar de que su uso en pacientes y personal médico ha ido en aumento. Dentro de los beneficios por su uso se han descrito el poder realizar seguimientos más objetivos y estandarizados, mayor comunicación entre los integrantes del equipo médico y respuestas más rápidas en casos de emergencias. Por parte de los pacientes estos han tenido mayor adquisición de conocimiento en relación a su enfermedad y mayores tasas de adherencia a los tratamientos.

Palabras clave: App, apps, mHealth, aplicación móvil, neurociencias.

\begin{abstract}
Thanks to the continuous development of telecommunications technologies, the use of smart phones has been increasing, favoring the growth of mobile applications (app). Currently there are more than 700,000 in the various platforms, of which $3 \%$ are related to health issues. Health apps are framed in the concept of mobile health or mHealth, which currently has no clear definition, but the WHO has referred to this as the practice of medicine and public health supported by mobile devices. So far there are several reviews on mobile applications in other medical disciplines, the amount of such reviews on apps being used in areas of neuroscience are few, very despite its use in patients and medical personnel has been increasing. Among the benefits of its use, it has been described being able to carry out more objective and standardized follow-ups, greater communication between the members of the medical team and faster responses in cases of emergencies. On the part of the patients, they have had greater knowledge acquisition in relation to their disease and higher adherence rates to the treatments.
\end{abstract}

Key words: App, apps, mHealth, mobile application, neurosciences. 


\section{Introducción}

El desarrollo de nuevas tecnologías ha generado una gran evolución en la telefonía móvil, favoreciendo la forma de cómo se accede a la información y a los servicios de salud ${ }^{1}$. Debido a la gran cobertura de los servicios móviles y al uso cotidiano de los smartphones (teléfonos inteligentes) han convertido a estas herramientas en un recurso con gran potencial, capaces de facilitar las conductas médicas ${ }^{2}$. Durante el año 2014 , el $80-96 \%$ de los médicos usaron su smartphone en su práctica ${ }^{3}$.

La unión internacional de telecomunicación (UIT), en su informe anual «Hechos y cifras de las tecnologías de la información y la comunicación en el mundo», estimó que al finalizar el año 2017 el uso de internet a través del smartphone superaría los 4.300 millones usuarios ${ }^{4}$, esto ha favorecido la difusión y globalización de las aplicaciones móviles entre la población general ${ }^{2}$.

Una aplicación móvil (app) es software que se instala en un dispositivo, generalmente smartphones o Tablet, el cual es capaz de ejecutar funciones para la cual ha sido diseñada ${ }^{1}$. Las apps de salud se encuentran enmarcadas en el concepto de salud móvil o mHealth, cuya definición en la actualidad sigue siendo poco clara ${ }^{2}$. La OMS (organización mundial para la salud) se ha referido como la práctica de la medicina y la salud pública soportada por dispositivos móviles como teléfonos móviles, dispositivos de monitorización de pacientes, asistentes personales digitales y otros dispositivos inalámbricos ${ }^{5}$.

Actualmente, existen más de 700.000 aplicaciones disponibles en las diversas plataformas, de las cuales el 3\% están relacionadas con salud ${ }^{5}$. Durante el año 2017 se reportaron 55.800 aplicaciones de salud en plataforma Apple IOS, de las cuales 159 correspondieron la especialidad de neurocirugía ${ }^{3}$. Las apps de salud buscan optimizar la comunicación médico-paciente, generar mayor apropiación de conocimiento por parte del paciente en relación a su patología, promoción de hábitos saludables y recolección de datos para facilitar la toma de decisión por parte del personal de salud ${ }^{6,7}$.

\section{mHealth y sus beneficios en la salud}

La OMS, en el año 2016, estimó que aproximadamente 1.000 millones de personas padecían algún trastorno neurológico ${ }^{2}$. Pradilla et al., en el año 2003, determinaron la prevalencia de 8 trastornos neurológicos en la población colombiana, reportando que por cada 1.000 personas el $71,2 \%$ padecía de migraña, $46,1 \%$ de trastornos del desarrollo neurológico, $19,9 \%$ de enfermedad cerebrovascular, $13,1 \%$ demencia, 10,3\% epilepsia, 8,5\% neuropatía periférica, y el 6,4\% tenía secuelas de traumatismo craneoencefálico ${ }^{8}$ (Tabla 1). Debido a estas cifras y a la evolución constante de las tecnologías de la información y comunicación (TIC), en la actualidad se están desarrollando nuevos sistemas que favorezcan el diagnóstico y tratamiento oportuno de patologías neurológicas, principalmente en epilepsia, demencia, enfermedad de Parkinson y accidentes cerebrovasculares $^{9-11}$. Patterson et al., en el año 2017, determinaron que el personal de la salud no médico de la india, que fue capacitado en el uso de una app capaz de identificar las crisis epilépticas, tuvieron tasas de diagnóstico erróneo similares a la de los médicos locales, demostrando así el gran potencial de las mhealth ${ }^{12}$.

Se han descrito diversos beneficios del uso de apps en el ámbito de la salud, a nivel del personal médico, facilita la realización de seguimientos más objetivos y estandarizados, disminuye las limitaciones geográficas y aumenta la interacción entre miembros de un mismo equipo ${ }^{10}$. Thapa et al., en el año 2016, implementaron un modelo compuesto por aplicaciones de uso gratuito en el departamento de neurocirugía de Kathmandu Medical College Teaching, logrando optimizar el manejo de los pacientes en el servicio de urgencias y una disminución en los costos ${ }^{13}$. Por otra parte, los pacientes son capaces de gestionar su salud al tener mayor información sobre la misma ${ }^{14}$ (Tabla 2).

\section{Consideraciones especiales frente al uso de aplicaciones móviles en neurociencias}

Por lo que se refiere al uso de aplicaciones móviles por parte del personal de salud, se han definido ciertas preocupaciones médico-legales y éticas que abarcan el uso indiscriminado de dichas aplicaciones, pudiendo llevar a desatender las quejas de los pacientes ${ }^{15,16}$, sumado a la intranquilidad que puede producir en los pacientes el uso de su información personal ${ }^{15}$. Por tal razón, la Food and Drug Administratión (FDA), para el año 2011, publicó un borrador que buscaba orientar sobre la reglamentación que se debía tener en cuenta a la hora de diseñar y crear aplicaciones con contenido médico ${ }^{16}$. Aunque la mayoría de aplicaciones aún no están certificadas debido a que la información dispuesta en estas resulta insuficiente para ofrecer ${ }^{11}$.
Tabla 1.

Prevalencia de las enfermedades neurológicas en Colombia ${ }^{8}$

\begin{tabular}{|l|c|}
\hline Migraña & $71,20 \%$ \\
\hline Trastornos del desarrollo neurológico & $46,1 \%$ \\
\hline Enfermedad cerebrovascular & $19,9 \%$ \\
\hline Demencia & $13,1 \%$ \\
\hline Epilepsia & $10,3 \%$ \\
\hline Neuropatía periférica & $8,5 \%$ \\
\hline Secuelas de traumatismo craneoencefálico & $6,4 \%$ \\
\hline *Porcentaje expresado por cada 1.000 personas. \\
\hline
\end{tabular}

\section{Aplicaciones disponibles en neurociencias}

A pesar de que hasta el momento existen varias revisiones sobre aplicaciones móviles (App) en otras disciplinas médicas, la cantidad de dichas revisiones sobre aplicaciones siendo utilizadas en áreas de la neurociencia son pocas ${ }^{15,16}$. Así pues, los resultados arrojados por un estudio en el año 2013, mostraron que existen aproximadamente 111 utilizadas en neurociencias ${ }^{15}$. Así mismo, el uso de aplicaciones móviles por 
Tabla 2.

Beneficios del uso de mHealth ${ }^{10,13,14}$

Personal médico

- Seguimientos médicos más objetivos

- Incrementa la colaboración entre los miembros del equipo médico

- Disminución de las limitaciones geográficas para la toma de conductas

- Disminución de errores médicos a través del uso de algoritmos digitales

Personal no médico

- Mayor conocimiento de su enfermedad

- Mayor adherencia a los tratamientos

- Mayor acceso a los servicios de salud

- Capacidad de autogestionar su enfermedad

Sistema de salud

- Disminución de costos

- Mejor calidad en la prestación de los servicios

- Disminución de consultas innecesarias

parte de médicos y estudiantes ha ido en aumento, y de igual forma se ha ido perfeccionando la idea hasta hacerla accesible y recomendable a los pacientes, con el objetivo de que estos últimos participen más en la prevención de sus enfermedades ${ }^{16,17}$.

Las aplicaciones comúnmente utilizadas han sido clasificadas de tal manera que permita la fácil comprensión de las funciones que ofrecen a las personas, ya sean médicos, estudiantes de medicina o pacientes ${ }^{15}$.

Entre las categorías encontradas tenemos: aplicaciones de herramientas clínicas, conferencias, educativas, literatura, marketing, de información para pacientes, y aquellas de referencias ${ }^{15}$. Sin embargo, otra forma de clasificarlas es aquellas dirigidas a médicos y dirigidas a la atención del paciente ${ }^{16}$.

Tomando el caso de las aplicaciones enfocadas en la educación, por ejemplo, el contenido temático abarca desde conceptos anatómicos, ciencias básicas, exámenes físicos y técnicas de cirugía, hasta conceptos radiológi$\cos ^{15}$. Además, la educación no solo tiene como meta a los profesionales en salud, sino que el propósito también es educar a los pacientes, familiares y cualquier otro que pueda influir sobre el cuidado de una enfermedad ${ }^{11,17}$.

Por el contrario, las apps que conforman el grupo que va enfocado a profesionales de la salud, ya sea que estén en formación o no, comprende todas aquellas aplicaciones de conferencias, artículos de investigación, libros académicos, y calculadoras médicas ${ }^{15,16}$. Ejemplo de esto, son aplicaciones como Skyscape, UpToDate, PudGet, entre otras ${ }^{16}$

De manera puntual, algunas de las aplicaciones existentes han concretado la información que ofrecen sobre patologías crónicas, en busca de mejorar el modo de vida de las personas que la padecen ${ }^{11}$. Así pues, se han diseñado aplicaciones que permiten a pacientes con epilepsia llevar un control de su medicación, como de sus convulsiones ${ }^{11,18}$. Además, se ha demostrado que los pacientes cuyas convulsiones diarias son más frecuentes, tienden a utilizar más las aplicaciones móviles que permitan llevar un control diario de sus convulsiones ${ }^{18}$. También estas aplicaciones han permitido que las personas con epilepsia mejoren, debido a la mayor información que tienen acerca de la enfermedad, lo que se evidencia en una recuperación al estrés que esta produce ${ }^{18,19}$.

Otras aplicaciones han ido más allá dentro del campo de las neurociencias, más específicamente neurocirugía y neuroradiología, cuyas funciones van desde la comunicación entre estudiantes y residentes de medicina, hasta imágenes radiológicas para simulación ${ }^{20,21}$. En particular, se ha diseñado una aplicación móvil y de computadora, cuya función es la de transmitir resultados radiológicos por imagen entre médicos en formación, permitiendo que la información llegue de forma breve y permita la toma de decisiones ${ }^{20}$. Ahora bien, en el caso de las aplicaciones para simulación, permiten que se perfeccionen técnicas de ejecución e interpretación de imágenes neuroradiológicas, que finalmente produzcan resultados satisfactorios sobre localización de lesiones que requieran ser llevadas a neurocirugía ${ }^{21}$.

\section{Conclusión}

Las aplicaciones móviles usadas en medicina o mHealth han servido a gran parte de la comunidad académica como herramientas de aprendizaje, estudio, práctica, comunicación, guía y control a pacientes. Teniendo en cuenta que actualmente la accesibilidad de médicos y pacientes a la telefonía inteligente y sus servicios es casi global, la puesta en marcha de dichas aplicaciones en medicina ha ido en aumento. Sin embargo, con tantas aplicaciones en el mercado, se ha visto en la necesidad de que instituciones intervengan y reglamenten estas con el fin de establecer qué información debe estar en manos de pacientes y qué información o herramienta hace falta para seguir apoyando a los médicos en su formación.

Recibido: 11 de diciembre de 2018 Aceptado: 26 de diciembre de 2018

\section{Referencias}

1. Santamaría-Puerto G, Hernández-Rincón E. Aplicaciones médicas móviles: Definiciones, beneficios y riesgos. Salud Uninorte. 2015;31(3):599-607.

2. Rodríguez MTS, Vázquez SC, Casas PM, Cuerda RC De. Apps en neurorrehabilitación. Una revisión sistemática de aplicaciones móvi- 
les. Neurología. 2018;33(5):313-26.

3. Bigder M, Dakson A, Elliott CA, Guha D, Lavergne P, Makarenko S, et al. Smartphone usage patterns by Canadian neurosurgery residents - A national cross- sectional survey. World Neurosurg. 2017;111(2018):465-75.

4. ITU. Resumen - Informe sobre la Medición de la Sociedad de la Información de 2017. 2017;8.

5. Alexander G, Puerto S, Hernando E, Rincón H. Aplicaciones de salud para móviles: Uso en pacientes de Medicina Interna en el Hospital Regional de Duitama, Boyacá, Colombia Use of mobile health applications with Internal Medicine patients at the Regional Hospital of Duitama, Boyacá, Colombia. Rev Cuba Inf en Ciencias la Salud. 2016;27(3):271-85.

6. World Health Organization. mSalud: uso de las tecnologías móviles inalámbricas en la salud pública. Informe de la Secretaría. 2016;1-4.

7. Sun J, Guo Y, Wang X, Zeng Q. mHealth For Aging China: Opportunities and Challenges. Aging Dis. 2016;7(1):53.

8. A GP, A BEV. Estudio neuroepidemiológico nacional (EPINEURO) colombiano. Rev Panam Salud Publica. 2003;14(2):104-11.

9. Konoike T, Iwashita K, Sasaki T, Hiraki K, Takahashi T, Papavassiliou GC, et al. Giant Shubnikov-de Haas oscillation in t-conductors. Synth Met. 2003;133-134(3):157-8.

10. Linares-del Rey M, Vela-Desojo L, Cano-de la Cuerda R. Aplicaciones móviles en la enfermedad de Parkinson: una revisión sistemática. Neurologia. 2016;1-17.

11. Le FA, Butler S, Beavis E, Gill D, Bye AME. EpApp: Development and evaluation of a smartphone/tablet app for adolescents with epilepsy. J Clin Neurosci. 2018;50:214-20.

12. Patterson V, Samant S, Bhushan M, Jain P, Agavane V, Jain Y. Diagnosis of epileptic seizures by community health workers using a mobile app: A comparison with physicians and a neurologist. Seizure Eur J Epilepsy. 2018;55:4-8.

13. Thapa A, Kc B, Shakya B. Cost Effective Use of Free-to-Use Apps in Neurosurgery (FAN) in Developing Countries: From Clinical Decision Making to Educational Courses, Strengthening Health Care Delivery. World Neurosurg. 2016;95:270-5.

14. Kroes N, Borg T. La salud en el bolsillo: la sanidad móvil despliega su potencial. Comun prensa Com Eur. 2014;

15. Zaki M, Drazin D. Smartphone use in neurosurgery? APP - solutely! 2014;

16. Nahed BV, Sheth KN. applications in neurology. 2013;52-61.

17. Investigation O. Visualization of Superficial Cerebral Lesions Using a Smartphone Application. 2018;28(3):349-55.

18. Liu X, Wang R, Zhou D, Hong Z. Smartphone applications for seizure care and management in children and adolescents with epilepsy: Feasibility and acceptability assessment among caregivers in China. Epilepsy Res. 2016;127:1-5.

19. Escoffery C, Mcgee R, Bidwell J, Sims C, Kovitch E, Frazier C, et al. Epilepsy \& Behavior A review of mobile apps for epilepsy selfmanagement. Epilepsy Behav. 2018;81:62-9.

20. Thapa A, Shrestha D, Shrestha D, Giri S. Use of viber app: A fast, easy and cost effective method of communication in neurosurgery. 2013;

21. Perin A, Prada FU, Moraldo M, Schiappacasse A, Galbiati TF, Riker NI, et al. USim: A New Device and App for Case-Specific, Intraoperative Ultrasound Simulation and Rehearsal in Neurosurgery. A Preliminary Study. 2017;0(0):1-7.

\section{Correspondencia a:}

Dr. Luis Rafael Moscote-Salazar

rafaelmoscote21@gmail.com 